\title{
Patterns of arousal in comatose patients
}

\author{
B. M. EVANS \\ From the Regional Department of Clinical Neurophysiology, \\ Brook General Hospital, London
}

SYNOPSIS The EEG changes, with associated cardiorespiratory phenomena and, where possible, postmortem findings, are described in six patients in coma, with some reference to other similar cases. Spontaneously occurring periodic changes were observed at varying intervals between $20 \mathrm{~s}$ and $4 \mathrm{~min}$ but most commonly at $\frac{1}{2}-2 \mathrm{~min}$. These involved simultaneous cardiorespiratory, EEG, and somatic motor phenomena related to changes in the level of arousal. It is suggested that a physiological periodicity of the arousal mechanisms exists which may be of importance in the understanding of the pathophysiology of comatose states.

Changes in the EEGs of comatose patients with associated changes in the pulse and respiration rates, related to arousal stimuli, were described by Fischgold and Mathis (1959) but have received little attention since. Periodicities of the same systems have also been observed in the early stages of sleep (Bulow, 1963, Lugaresi et al., 1972) and in subacute spongiform encephalopathy (Evans, 1975).

Changes of similar timing have been widely studied in the cerebrospinal fluid pressure which are also associated with cardiorespiratory, blood pressure, and EEG changes (Lundberg, 1960; Ingvar and Lundberg, 1961; Kjallquist et al., 1964; Cooper and Hulme, 1966; Symon et al., 1972).

This study presents some of the results obtained from monitoring a large number of patients in coma. Those patients in whom the changes were most prominent or instructive have been described in detail. It is suggested that all the different periodicities mentioned have a common origin in the inherent periodicity of the arousal mechanisms, the system in which the changes are most apparent being determined by the particular pathological or physiological circumstances.

\section{METHODS}

All the patients studied were admissions to the emergency or neurosurgical services of the Brook (Accepted 16 December 1975.)
General Hospital, London. The records were made with SLE encephalographs, E18/16 or E8b, using silver disc or needle electrodes placed according to the modified Maudsley system (Pampiglione, 1956). The electrocardiogram (ECG) was recorded from the left and right shoulder, giving a modified standard lead 1 . The respiration was recorded with a thermocouple transducer over the nose or mouth.

The respiratory and pulse rates were calculated from the paper speed by measuring each breath from a suitable stable feature on the trace and between every other $\mathrm{R}$ wave on the ECG. Line graphs were then drawn to show the changes of rate at $2 \mathrm{~s}$ intervals. In each of the Figures these graphs have been shown under a representative section of the EEG, the location of which has been indicated on the graphs by broad arrows. If the machine was stopped, the base line of the graph has been broken. If EEG changes could be seen clearly, their duration has been shown by a solid line above the graphs; in each case the respiration graph has been shown above the pulse rate graph but the time scale is the same for both and they were plotted concurrently. Other notations are described individually and, where possible, the neuropathology is illustrated on the Figures.

\section{CASE HISTORIES AND EEG FINDINGS}

\section{CASE 1}

$(N 28438 M B)$ A woman aged 53 years whose previous health was good, apart from menstrual irregularities which had been treated with oestrogens for three years, collapsed unconscious in the street. Admitted to her local hospital, she was found to be 


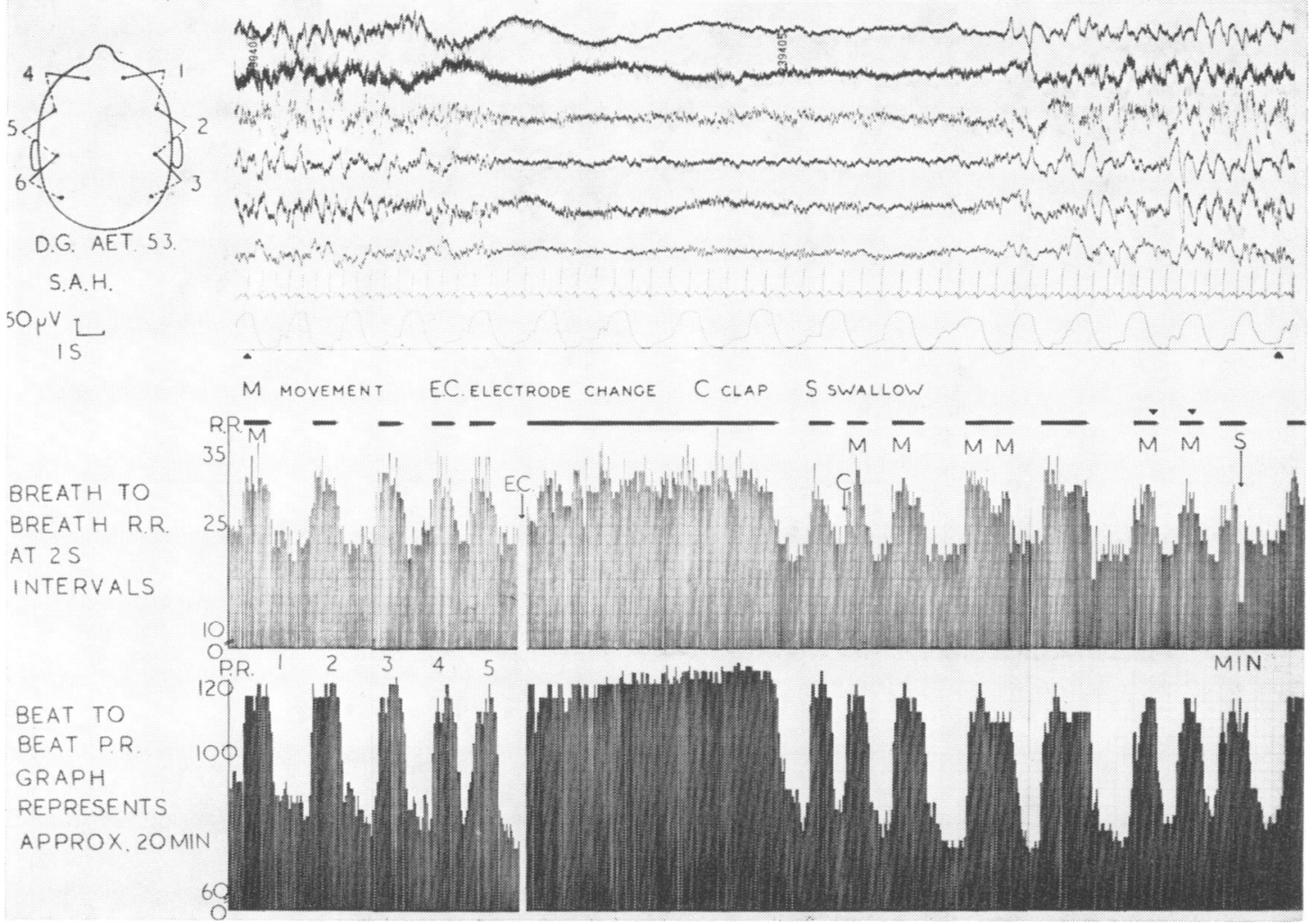

FIG. 1 Case 1. Woman, 53 years, subarachnoid haemorrhage. Above: EEG at $1.5 \mathrm{~cm} / \mathrm{s}$. Bursts of 1-7 Hz activity alternating with lower voltage 7-12 Hz activity. Pulse and respiratory rate change in relation to EEG pattern. Below: graphs of breath to breath respiratory rate and beat to beat pulse rate at $2 \mathrm{~s}$ intervals. Solid black line indicates periods of EEG change. Events occurring at one to two minute intervals, event at $\mathrm{C}$ precipitated by clap. Prolonged episode of raised pulse and respiration after electrode change (E.C.).

deeply unconscious with a right hemiplegia and a blood pressure of $210 / 130 \mathrm{mmHg}$. The CSF was uniformly blood stained. During the following three days her level of consciousness improved and she was transferred to the neurosurgical unit. On admission, she was drowsy and dysphasic with a dense right hemiplegia sparing the face. She had subhyaloid haemorrhages in both eyes and her blood pressure was $150 / 90 \mathrm{mmHg}$. Left carotid angiography showed no aneurysm but displacement of the pericallosal artery and the deep veins to the right suggested a small intracerebral clot. Three days after admission her level of consciousness deteriorated and she died after an illness of eight days. Postmortem examination was refused.

The EEG (Fig. 1) was recorded four days before her death; she was very drowsy but occasionally obeyed simple commands; her pupils were equal and reacted to light. The record showed frequent prolonged bursts of high voltage activity at frequencies between 1 and $7 \mathrm{~Hz}$ alternating with periods of low voltage activity at frequencies between 7 and $12 \mathrm{~Hz}$. Each episode of slow activity was associated with an increase in the pulse and respiration rate. The end of each episode of slow activity showed marked 'swinging' of the frontal channels probably due to rolling eye movements (Fig. 1). The pulse and respiratory rate graph below the EEG record showed that the bursts of slow activity were occurring at one to two minute intervals, most commonly at about one minute and that some of them were associated with movements of the limbs or head, or by swallowing, chewing or sighing. Any minor stimulus, pinch, clap, or even a loud noise on the ward occurring when the EEG was of 


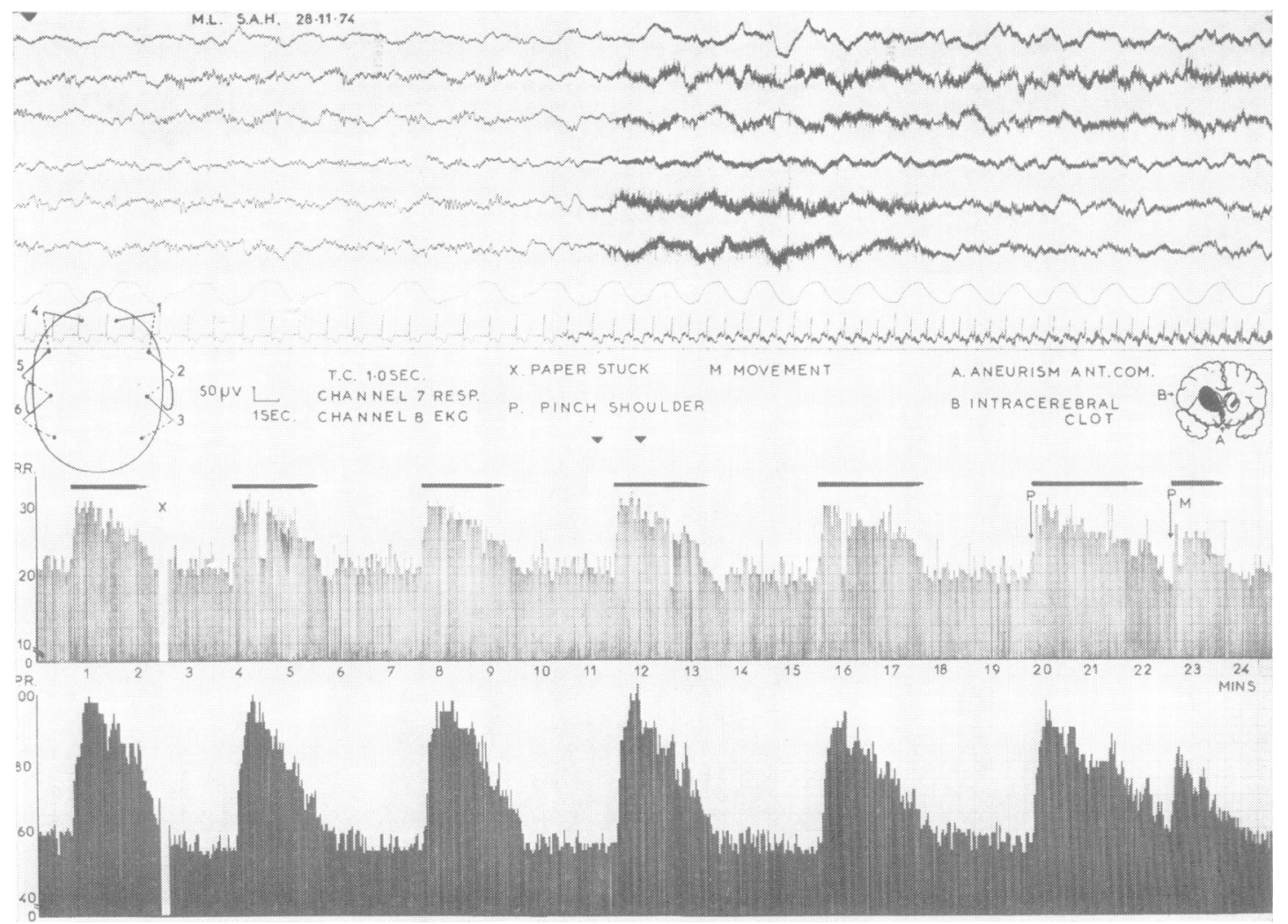

FIG. 2 Case 2. Woman, 66 years, subarachnoid haemorrhage. Anterior communicating artery aneurysm. Above: EEG at $1.5 \mathrm{~cm} / \mathrm{s}$, low voltage $7-10 \mathrm{~Hz}$ and slower waves followed by suppression and then higher voltage slow waves with muscle artefact. Increase in pulse and respiratory rates start with suppression. Below: events occurring at about four minute intervals. Last event precipitated by pinch $(\mathrm{P})$ and further peak by second pinch. Indication of faster $20 \mathrm{~s}$ interval on descending pulse rate graph at 12 th and 16 th minutes. EKG=electrocardiogram.

low voltage would precipitate slow wave activity which was identical with that occurring spontaneously. This effect is indicated at $\mathrm{C}$ on the graph in Fig. 1.

At one point an electrode was changed (E.C. on Fig. 1) and this was followed by a period of the EEG slow activity with raised pulse and respiration rates which lasted for nearly five minutes before subsiding spontaneously. During this period the patient remained motionless.

Changes similar to those described have been observed in many other patients with subarachnoid haemorrhage, although not always so well marked.

\section{CASE 2}

$(N 28559 B)$ A woman of 66 years with angina pectoris suddenly developed severe occipital headache and lost consciousness briefly. Headache persisted and her CSF was bloodstained with xanthochromic supernatant fluid. She was transferred to the neurosurgical unit and found to be alert with a slight right facial weakness and a blood pressure of 130/70 $\mathrm{mmHg}$. Bilateral angiography revealed an aneurysm of the anterior communicating artery which was judged to be inoperable. Thirteen days after the initial haemorrhage she became unconscious with a right hemiplegia; she died after an illness of five weeks. At postmortem examination the heart showed marked ventricular hypertrophy and fibrosis. The aneurysm of the anterior communicating artery was associated with a left intracranial haemorrhage extending from the frontal pole to the anterior end of the corpus striatum; microscopy of the haemor- 


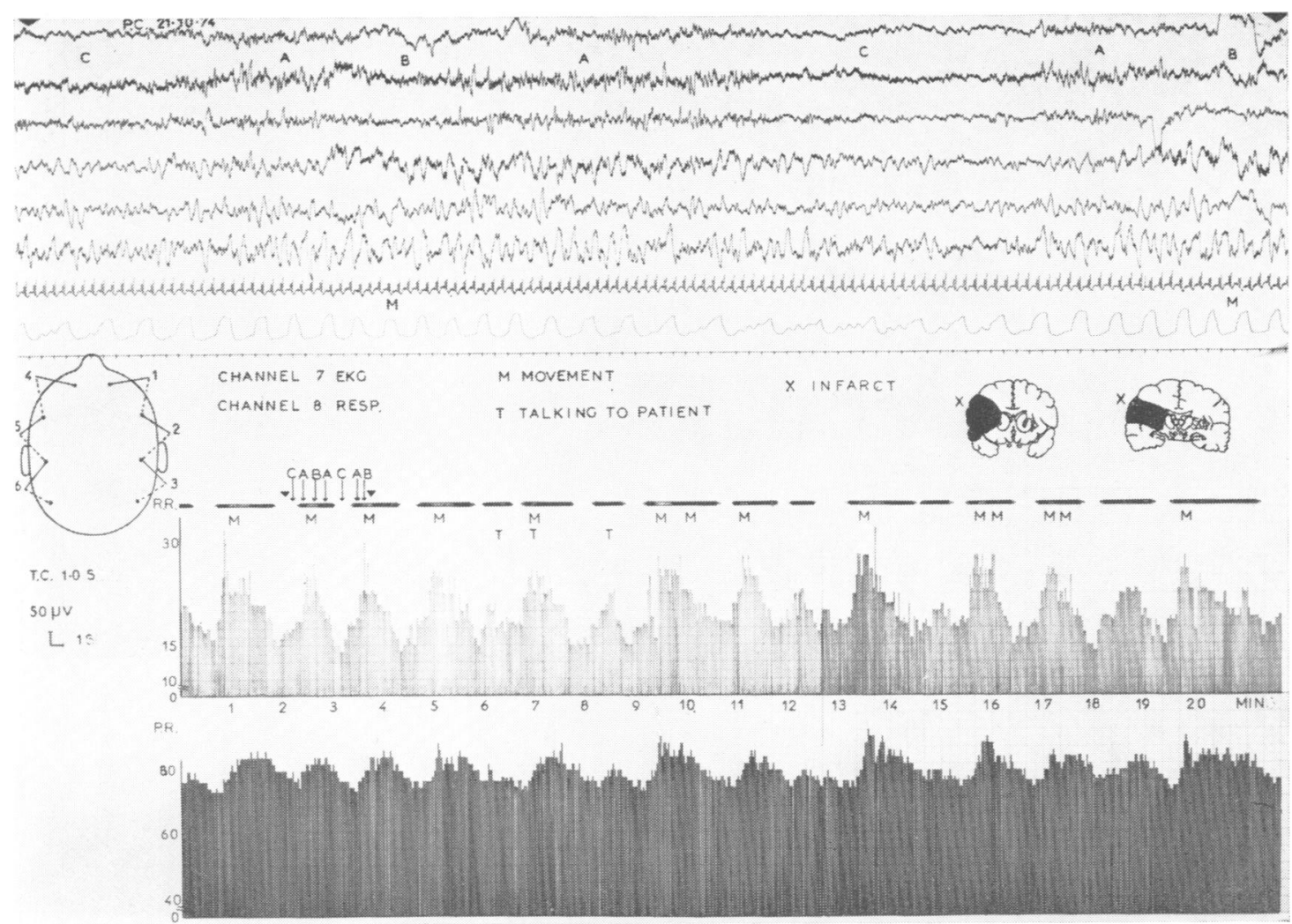

FIG. 3 Case 3. Woman, 42 years, left middle cerebral artery embolus. Above: EEG at $0.75 \mathrm{~cm} / \mathrm{s}$. Channels I, 2 , and 3 right hemisphere $2-3 \mathrm{~Hz}$ waves of higher voltage (A) alternate with $6 \mathrm{~Hz}$ theta $(\mathrm{B}$ and $\mathrm{C}), \mathrm{C}$ periods associated with slower pulse and respiration, $B$ periods associated with movement and high pulse and respiration rates. Channels 4, 5, and 6 left hemisphere. Continuous very slow waves during $A$ and B periods of lower voltage during $C$ periods. EKG=electrocardiogram. Below: events at about one minute intervals. Some irregularity when spoken to $(\mathrm{T})$.

rhage showed it to have been present for more than 72 hours. It involved the anterior part of the caudate nucleus and the anterior limb of the corpus callosum; there was also some herniation of the cingulate gyrus (Fig. 2). Blood was present in the left ventricle but there was no fresh subarachnoid harmorrhage.

The EEG was taken three days after her final deterioration. She was responding to painful stimuli with quasipurposeful movements in her right limbs only; her pupils were equal and reacted to light. Her blood pressure was $190 / 90 \mathrm{mmHg}$. The record showed spontaneous periodic changes; low voltage $7-10 \mathrm{~Hz}$ activity mixed with some slower waves at about $1 \mathrm{~Hz}$ was interrupted by a suppression with loss of the faster frequencies followed by higher voltage slow waves. During the latter period, the patient shivered slightly and muscle artefact appeared on the trace (Fig. 2). At the time of the suppression there was an increase of pulse and respiration rates which persisted for 1-2 min.

The graphs in Fig. 2 showed that the events were occurring at about four minute intervals and that they were sensitive to stimulation. The last event, at 20 minutes on the graph, was precipitated by a pinch and a further pinch given when the EEG change had subsided produced a secondary peak. The pulse rate graph showed some evidence of a shorter interval at about $20 \mathrm{~s}$. This is best seen at the 12th and 16th minutes on the graph, during the fall of the pulse rate after the initial rise.

Very similar changes were seen in this patient in a 


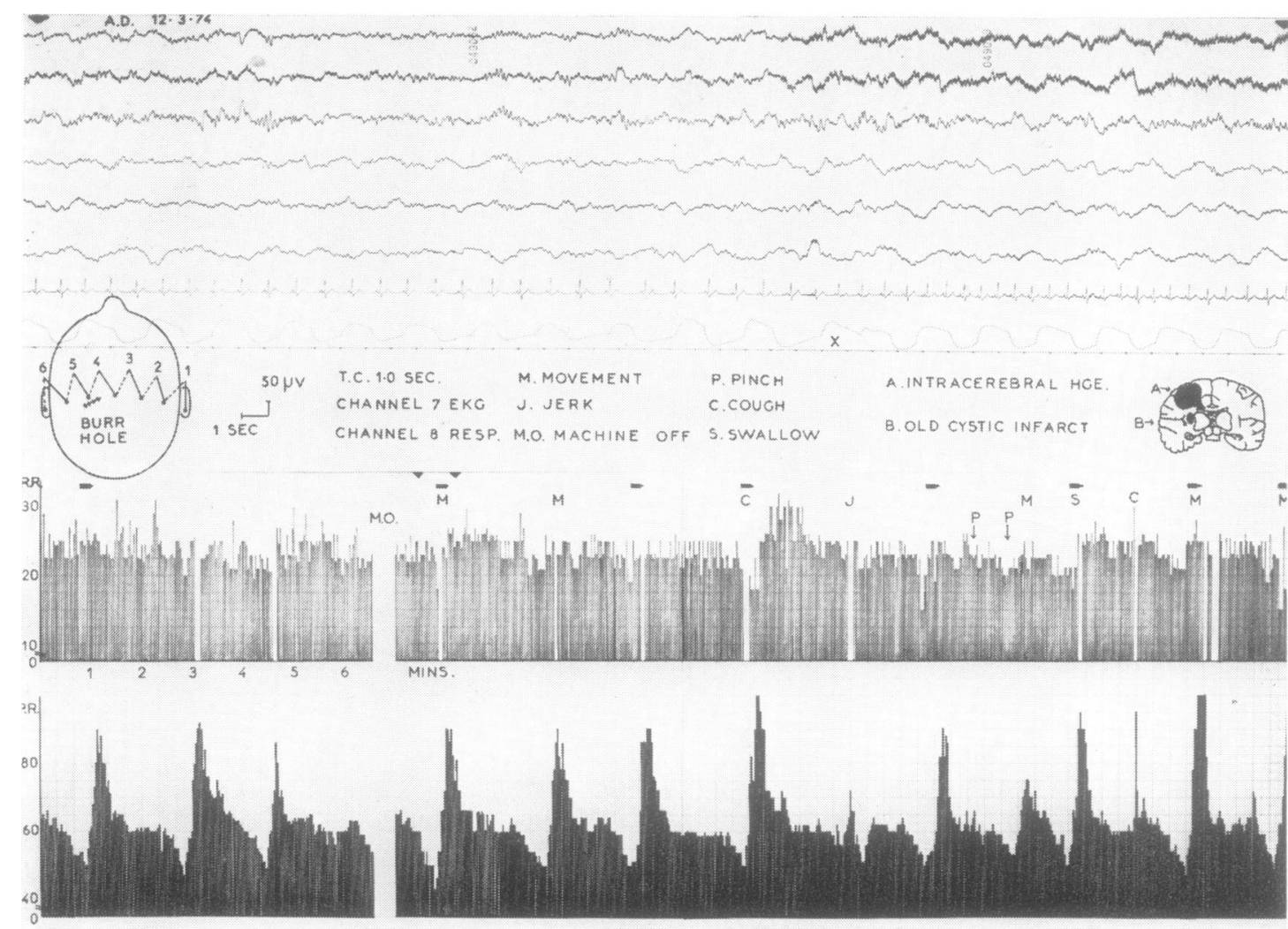

FIG. 4 Case 4. Man, 49 years. Left intracerebral haemorrhage and old infarct. Above: EEG at $1.5 \mathrm{~cm} / \mathrm{s}$. Slowest activity and loss of faster frequencies over left hemisphere. Minimal EEG change at time of pulse rate increase and double breath (X). Below: events at about two minute intervals. Short gaps on respiration graph indicate double breaths. EKG=electrocardiogram.

record taken two days earlier, they were absent from a third record two days before death.

\section{CASE 3}

(227122) A woman aged 42 years had been noticed to have a cardiac murmur 19 years previously and had been breathless for three years. She was admitted to hospital with a history of sudden onset of inability to move her right arm and leg or to speak, coupled with severe dyspnoea. On admission, she was unconscious with a profound right hemiplegia involving the face, and acute pulmonary oedema. She was treated with diuretics, aminophylline, diamorphine, and cedilanid. An hour later she had a respiratory arrest lasting for eight minutes which recovered spontaneously; her heart rate remained steady at $80 / \mathrm{min}$.
A diagnosis was made of mitral stenosis with a cerebral embolus; blood cultures were negative. The following day she was conscious but drowsy, looking around and making purposeful movements with her right arm; she did not speak or obey commands because of her dysphasia. She had a dense right hemiplegia involving the face and both plantar responses were extensor. She remained in about the same condition with a regular pulse between 80 and 100 per min until she died during the night after an illness of six days.

Postmortem examination showed a massive left hemisphere infarct involving the territory of the middle cerebral artery. It extended from the frontal pole to the parietal regions and involved the tip of the temporal lobe. It involved the head of the caudate nucleus but spared the thalamus. There were no other lesions in the brain. The heart showed mitral 
stenosis with fusion of the cusps; there was no evidence of bacterial endocarditis.

The EEG in Fig. 3 was taken 36 hours before death. The top three channels from the right hemisphere showed varied activity. Periods of low voltage, $6 \mathrm{~Hz}$ activity (labelled $\mathrm{B}$ and $\mathrm{C}$ ) alternated with higher voltage waves at $2 \frac{1}{2}-3 \mathrm{~Hz}$ (labelled $\mathrm{A}$ ). There was periodicity of the respiration. The slower respiratory rates were associated with the lower voltage $C$ periods, during which the patient was lying still with her eyes closed, whereas the highest rates were associated with the $B$ periods when the patient often made a purposeful movement or opened her eyes. The traces from the left hemisphere (channels 4, 5, and 6) showed continuous slow waves which were of lower voltage during the $C$ periods than at other times. The graphs showed the events occurring at about one minute intervals, when the patient was indisturbed; talking to her produced some irregularity. From the EEG alone it would have been difficult to distinguish between the $B$ periods and the $\mathrm{C}$ periods, although they clearly represented different clinical situations.

\section{CASE 4}

(28780B) A man of 49 years developed dysphasia and right-sided weakness two weeks before transfer to the neurosurgical unit. He had had a thoracotomy one year before to remove a tumour from his right lung which proved to be a secondary melanoma. On admission, he was alert but dysphasic with a right hemiparesis. His condition deteriorated rapidly. Carotid angiography showed a large avascular mass in the right parietal region. A biopsy of the area produced pigmented cells thought to be a secondary carcinoma.

At postmortem examination a melanomatous deposit was found in the head of the pancreas, but in the brain there was no evidence of tumour either macroscopically or microscopically. A large intracerebral haemorrhage occupied the left frontoparietal region extending into the corpus callosum but not involving the basal nuclei. There was an old cystic infarct of the internal capsule on the left side.

The EEG in Fig. 4 was taken the day before death. He was responding by quasipurposeful movement with his left limbs to painful stimuli. He had equal pupils reactive to light and a blood pressure of $130 / 80 \mathrm{mmHg}$. The traces showed low voltage $1.0 \mathrm{~Hz}$ activity over both sides most marked on the left with loss of faster frequencies on the left. Spontaneous arousals occurred, with swallowing, coughing, and chewing, which were associated with slightly higher voltage activity over the right hemisphere and with a marked increase in the pulse rate. Marked fluctua- tions of the pulse rate were also seen which were not associated with clinical arousals. The pulse rate graph showed a stereotyped pattern, sharp peaks at about two minute intervals were followed by a plateau and then by a dip before the next peak.

The respiration showed little periodicity but a double breath marked X on Fig. 4 was seen with each clinical arousal or increase in pulse rate. This breath has not been charted on the respiratory graph and appears as a gap which can be seen to coincide with the pulse rate peaks, whether or not these were associated with a clinical arousal (see third and fourth minutes of graph on Fig. 4).

\section{CASE 5}

$(28486 N)$ This 57 year old woman was admitted with a four week history of gradually increasing weakness and sensory disturbance of the right limbs, with headache. On admission, she was conscious and orientated with a left hemiparesis and sensory loss of cortical type. Her level of consciousness rapidly deteriorated until she was responding only to painful stimuli. A right carotid angiogram showed a large avascular mass in the right frontal and anterior parietal regions. Burr hole biopsy revealed a necrotic, malignant glioma. She died after an illness of four weeks. At postmortem examination there was a large glioma of the right frontoparietaloccipital region measuring $7 \mathrm{~cm}$ by $5 \mathrm{~cm}$, extending from the head of the caudate nucleus to the level of the splenium. There was marked cingulate herniation, uncal grooving and compression of the mid brain with no reactionary haemorrhage.

The EEG in Fig. 5 was taken the day before the patient died. She was very drowsy but responded purposefully to painful stimuli. Her pupils were equal and reacted to light. She made frequent, spontaneous flexion movements mainly with her right limbs. Her blood pressure was $140 / 80 \mathrm{mmHg}$.

The record showed little evidence of the underlying tumour; the principal feature was high voltage slow waves at $1-3 \mathrm{~Hz}$, maximal anteriorly. There was little periodicity of the respiration but the pulse rate showed marked fluctuations often associated with spontaneous movement. At periods of slower pulse rate there was some lowering of the EEG voltage. The pulse rate graph showed that the principal events were occurring at $\frac{1}{2}-1 \frac{1}{2}$ minute intervals but that these peaks were associated with subsidiary changes at shorter $20 \mathrm{~s}$ intervals (see events between one and two minutes and again between 17 and 18 minutes on graph Fig. 5). The period between the 11th and 16th minutes was taken up by a prolonged episode of raised pulse rate with the shorter $20 \mathrm{~s}$ interval superimposed throughout. 

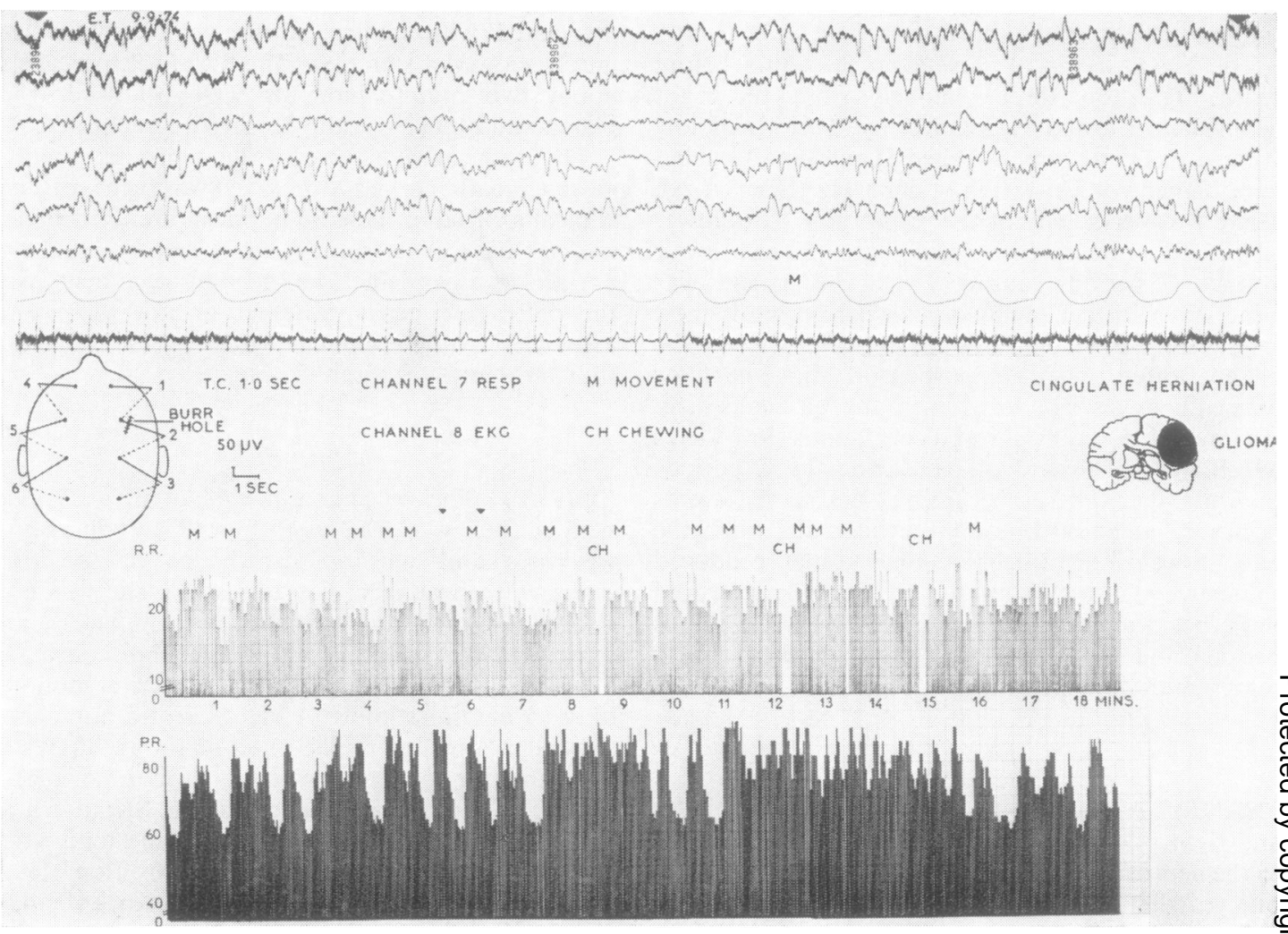

FIG. 5 Case 5. Woman, 57 years. Grade 11 astrocytoma. Above: EEG at $1.5 \mathrm{~cm} / \mathrm{s}$. Continuous high voltage slow activity maximal frontally. Some variation in pulse rate little respiratory change. Below: pulse rate graph shows major peaks at $\frac{1}{2}-1 \frac{1}{2}$ minutes with evidence of secondary peaks at $20 \mathrm{~s}$ best seen on events between first and second minutes and 17th and 18th minutes. Also during episode of raised pulse rate between 11th and 16th minutes. $E K G=$ electrocardiogram.

\section{CASE 6}

$(N 29016 M B)$ This 36 year old man was admitted to hospital after a road traffic accident without loss of consciousness. He was alert, with fractures of the shaft of the left femur, the left ischiopubic ramus, and the sixth and seventh ribs. The following day he became semiconscious with a blood pressure of $100 / 50 \mathrm{mmHg}$ and pulse rate of $130 / \mathrm{min}$. He was given $540 \mathrm{ml}$ blood and $540 \mathrm{ml}$ plasma and briefly improved. His level of consciousness again deteriorated, with dyspnoea and hyperventilation; a left haemothorax was drained before transferring him to the neurosurgical unit. On admission he was responding to stimulation with decerebrate movements. It was concluded that he was severely hypoxic due to blood loss and whole blood was therefore given as quickly as possible, with considerable general improvement. During the next five days he showed frequent, violent, spontaneous, decerebrate spasms. These gradually lessened with steadily improving level of consciousness. He eventually made an excellent recovery and returned to work four months after the accident.

The EEG shown in Fig. 6 was taken 36 hours after admission. At this time the patient was having frequent spasms both spontaneously and on minimal stimulation, a light touch or loud noise being sufficient to precipitate one. Each spasm consisted of extension of the back and neck with extension and internal rotation of the arms and legs and was associated with sweating and dilatation of the pupils. He showed no awareness of his surroundings. There were no focal signs in the nervous system. With each spasm the EEG showed high voltage activity at 1.0 


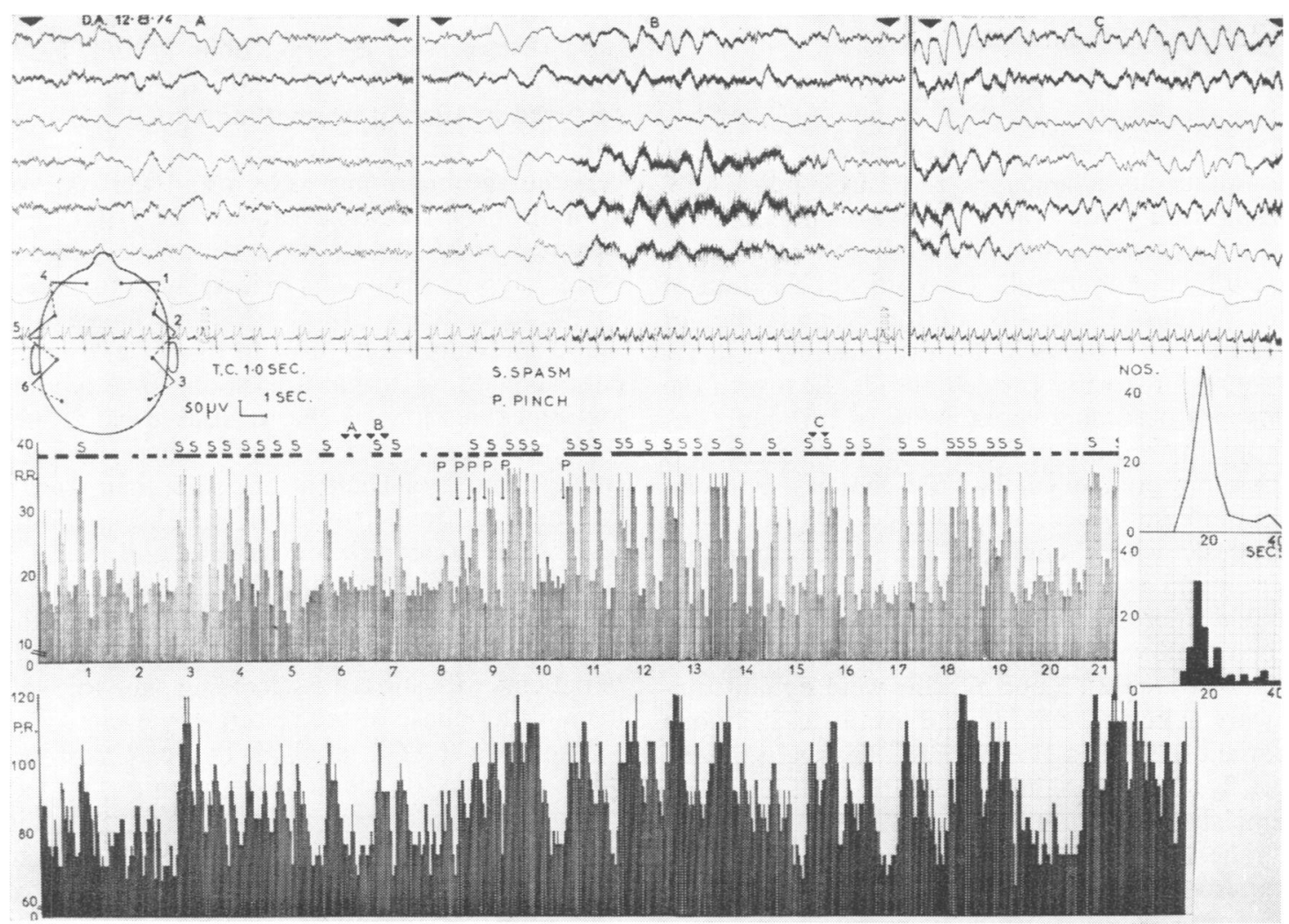

FIG. 6 Case 6. Man, 36 years. Decerebrate spasms. ?Aetiology. Above: EEG at $1.5 \mathrm{~cm} / \mathrm{s}$. A. EEG shows slow waves without spasm but some pulse and respiration change. B. Slow wave burst with spasm and marked pulse and respiration increase. C. Taken between two spasms, EEG remains slow but pulse and respiratory rate decrease. Below: respiration peaks at approximately $20 \mathrm{~s}$ intervals, see histogram and curve. Periods of relative freedom from spasms (S) at second, eighth, and 20th minutes alternate with frequent spasms and continuous EEG slow activity. $\mathrm{P}$ shows effects of pinch.

$\mathrm{Hz}$ or less and there was a marked increase in the pulse and respiration rates. Between the spasms the EEG activity was at $8-10 \mathrm{~Hz}$ of low voltage (Fig. 6B). The spasms tended to occur in groups with periods of relative freedom between. During the more quiescent times the EEG showed bursts of slow waves, with some increase in pulse and respiration rates, which were not accompanied by spasms (Fig. 6A). During the times when the spasms were frequent the EEG continued to show high voltage slow waves between the spasms, even when the pulse and respiration rates had dropped (Fig. 6C; taken between spasms). The pulse and respiration graphs showed that the spasms were occurring at about $20 \mathrm{~s}$ intervals and that respiratory and pulse rate peaks were seen at the same intervals during the periods without spasms (see second, eighth, and 20th minutes of graph). The histogram in Fig. 6 demonstrates the preferred $20 \mathrm{~s}$ interval; it shows the results of measurements between all the respiratory peaks during one hour of record. The curve above shows the number of intervals in any $4 \mathrm{~s}$ period. A series of pinches (P, Fig. 6) were given when the EEG showed $8-10 \mathrm{~Hz}$ activity and the patient was still. The first pair of pinches produced only EEG change; the second pair isolated spasms and the third pair a series of spasms.

There is some doubt about the exact aetiology of the cerebral disturbance in this patient; however, the clinical picture of frequent, highly reactive decerebrate spasms is commonly seen in children with head injuries. Similar data have been collected from a 
seven year old child in whom the spasms were also occurring at $20 \mathrm{~s}$ intervals.

\section{DISCUSSION}

The events observed in these patients consist of simultaneous changes in the EEG, pulse rate, respiration rate, and somatic musculature which occur spontaneously but can be precipitated by minor stimuli. The clinical behaviour of the patient at the time of the event is that of arousal and the stimuli produce arousal responses in the EEG which are identical with the changes occurring spontaneously, although differing in appearance from patient to patient. It is therefore concluded that the changes represent spontaneously occurring changes in the level of arousal, which confirms the observations of Fischgold and Mathis (1959).

The increase in arousal was not necessarily correlated with greater awareness, a point illustrated by cases 2 and 6 who were equally unaware in periods of high and low arousal. In one patient (case 3) a further EEG change was seen briefly at the height of each arousal at which time she was aware of her surroundings.

The interval between the spontaneous events varies considerably from case to case, the most common interval being between $\frac{1}{2}$ and two minutes. In some patients the intervals were much longer (case 2) and in some shorter at about 20 s (case 6). Cases 2 and 5 suggest that the shorter interval may be present at the same time as the longer one.

It is probable that the triggering effect of arousal stimuli is mediated by the same mechanisms as those responsible for the orienting, startle, and defensive reflexes originally described by Pavlov in 1927, Strauss in 1929, and Sokolov in 1960. These reflexes arise in response to any novel stimulus and involve the same systems as those described here (EEG, heart rate, respiration rate, and somatic musculature), as well as the skin resistance and pupil size. They habituate rapidly in the alert state but fail to do so during sleep (Sokolov and Paramanova, 1961 ; Johnson and Lubin, 1967). In fact, there may be some augmentation of the heart rate response in sleep (Johnson and Lubin, 1967).

OTHER PERIODICITIES RELATED TO CENTRAL NERVOUS SYSTEM Periodic respiration has been recognized in the early stages of sleep for a long time (Magnussen, 1944; Robin et al., 1957; Bulow, 1963). Bulow (1963) showed that it occurred in $10 \%$ of normal individuals; he also commented on the extremely close relationship between the breathing pattern and the state of arousal of the EEG. Coccagna et al. (1971) and Lugaresi et al. (1972) have shown respiratory periodicity in sleep which is related to corresponding changes of the skin resistance and blood pressure and also to limb jerking in patients with nocturnal myoclonus. The physiological expression of the arousal cycle would therefore seem to be the early stages of sleep (stage 1 and 2 of Dement and Kleitman, 1957). North and Jennett (1974) studied periodic respiration in acute brain damage and found it to be present in 55 of their 227 cases. Poole (1960) has shown a correlation between the periodic slow wave discharge in the EEG records of patients with subacute sclerosing panencephalitis and the phase of the respiratory cycle.

Lundberg (1960) studied the cerebrospinal fluid pressure by continuous monitoring. He described several types of short-term variation in pressure which show a very close resemblance to 8 the periodicities of this series. He recognized three different phenomena which he called the $\mathrm{A}, \mathrm{B}$, and $\mathrm{C}$ waves. The $\mathrm{B}$ waves occurred at intervals of $\frac{1}{2}$ to two minutes, were seen at both high and normal pressures but were present only in sleep or coma and not when the patient was alert. They were always accompanied by periodic respiration and sometimes by movements of the patient, more rarely they were associated with myoclonus. Kjallquist et al. (1964) and Cooper and Hulme (1966) have shown that these waves are associated with changes in the blood pressure and pulse rate. These $B$ waves bear a striking resemblance to the $\frac{1}{2}$ to two minute waves of the present series.

The A waves of Lundberg, also known as plateau waves, are more prolonged episodes of raised pressure lasting several minutes, which usually occur spontaneously but are sometimes precipitated by minor arousal stimuli. They are seen both in alert and comatose states but are always associated with raised intracranial pressure. These waves are probably the same phenomenon as the prolonged periods of raised pulse and respiration rates shown by cases 1 and 
5. The latter patient certainly had raised pressure and the former probably had. Ingvar and Lundberg (1961) performed EEGs on those patients in their series who had myoclonic jerks and found no paroxysmal activity but only slight but definite increase in arousal.

The $\mathrm{C}$ waves of Lundberg are a shorter phenomenon seen four to six times a minute and are often seen superimposed on the $A$ waves in much the same way that the shorter $20 \mathrm{~s}$ interval was seen during the period of raised pulse rate in case 5 .

Cooper and Hulme (1966) studied the CSF pressure continuously with a cortical pressure transducer and observed the same fluctuations particularly during stage 1 and 2 of sleep but absent from deeper levels of sleep as well as from the alert state.

Another system in which periodicities have been observed is the systemic blood pressure. Waves known as the Traub-Herring-Meyer waves were originally described in 1865 . They occur about four to six times a minute and were believed by Coccagna et al. (1971) and Lugaresi et al. (1972) to be related to the periodic events they recorded in sleep and by Lundberg (1960) to be related to the $C$ waves in the CSF pressure. The present study suggests that they may also be related to the timing of events in some patients with decerebrate spasms, a hypothesis that receives some support from the observation of Barnes and Burnham (1969) that each Mayer wave is preceded by an increase in excitability of the monosynaptic lumbar reflex.

ORIGIN OF THE CHANGES Although arousal is clearly a neural phenomenon associated with the reticular system, it is not necessarily the case that the primary mechanism is neural in origin. It is possible that changes in cerebral blood flow, blood pressure, $\mathrm{pH}$ or $\mathrm{CO}_{2}$, about which no information is available in this study, are giving rise to secondary changes in the reticular system. A reciprocal relationship between the systemic blood pressure and the reticular system has been demonstrated experimentally by Baust et al. (1963) and by Kakolewski and Takeo (1967) and a direct effect on cerebral blood flow by the reticular activating system has been shown experimentally by Meyer et al. (1969) and clinically by Ingvar et al. (1964).

It is probably fruitless to attempt an exhaustive discussion in the face of inadequate data, however this series lends some support to the hypothesis that central nervous system periodicities may be due to an inherent brain-stem rhythm. This possibility was suggested by Kjallquist et al. (1973) to explain the periodic CSF pressure changes and by Lugaresi et al. (1972) to explain the periodicities of sleep.

Whatever may be the theoretical basis of these phenomena, their practical implications may be more relevant. For instance, it is difficult to imagine a situation more likely to lead to trouble after a subarachnoid haemorrhage from an aneurysm than a cardiovascular system which behaves as though the patient were performing a 50 yard dash every time he stirs in bed.

Mechanisms which are related to changes in so many areas, both within the cranium and elsewhere, must be of importance in comatose states and also possibly in many other conditions.

\section{REFERENCES}

Barnes, C. D., and Burnham, E. (1969). The reflection of third order blood pressure waves in the lumbar monosynaptic reflex. Brain Research, 13, 183-186.

Baust, W., Niemczyk, H., and Vieth, J. (1963). The action of blood pressure on the ascending reticular activating system with special reference to adrenaline-induced EEG arousal. Electroencephalography and Clinical Neurophysiology, 15, 63-72.

Bulow, K. (1963). Respiration and wakefulness in man. Acta Physiologica Scandinavica, 59, suppl. 209.

Coccagna, G., Mantovani, M., Brignani, F., Manzini, A., and Lugaresi, E. (1971). Arterial pressure changes during spontaneous sleep in man. Electroencephalography and Clinical Neurophysiology, 31, 277-281.

Cooper, R., and Hulme, H. (1966). Intracranial pressure and related phenomena during sleep. Journal of Neurology, Neurosurgery, and Psychiatry, 29, 564-570.

Dement, W. C., and Kleitman, N. (1957). Cyclic variations in EEG during sleep and their relation to eye movements, body motility and dreaming. Electroencephalography and Clinical Neurophysiology, 9, 673-690.

Evans, B. M. (1975). Cyclic EEG changes in subacute spongiform and anoxic encephalopathy. Electroencephalography and Clinical Neurophysiology, 39, 587-598.

Fischgold, F., and Mathis, H. (1959). Obnubilations, comas et stupeurs. Electroencephalography and Clinical Neurophysiology, 11, suppl.

Ingvar, D., Haggendal, E., Nilsson, N. J., Sowrander, P., Wicklsom, I., and Lessan, N. A. (1964). Cerebral circulation and metabolism in a comatose patient. Archives of Neurology, 11, 13-21.

Ingvar, D. H., and Lundberg, N. (1961). Paroxysmal symptoms in intracranial hypertension studied with ventricular fluid pressure recording and EEG. Brain, 84, 446-459. 
Johnson, L. C., and Lubin, A. (1967). The orienting reflex during waking and sleeping. Electroencephalography and Clinical Neurophysiology, 22, 11-21.

Kakolewski, J. W., and Takeo, Y. (1967). Relationships between EEG patterns and arterial pressure changes. Electroencephalography and Clinical Neurophysiology, 22, 239-244.

Kjallquist, Å., Lundberg, N., and Ponten, U. (1964). Cardiovascular changes during rapid spontaneous variations of ventricular fluid pressure in patients with intracranial hypertension. Acta Neurologica Scandinavica, 40, 291-317.

Lugaresi, E., Coccagna, J., Mantovani, M., and Lebrun, R. (1972). Some periodic phenomena arising during drowsiness and sleep. Electroencephalography and Clinical Neurophysiology, 32, 701-705.

Lundberg, N. (1960). Continuous recording and control of ventricular pressure in neurosurgical practice. Acta Psychiatrica Scandinavica, suppl. 149, 36.

Magnussen, G. (1944). Studies on the Respiration During Sleep. A Contribution to the Physiology of Sleep Function. Lewis: London.

Meyer, J. S., Normura, F., Sakamoto, K., and Kondo, A. (1969). Effect of stimulation of the brain-stem reticular formation on cerebral blood flow and oxygen consumption. Electroencephalography and Clinical Neurophysiology, 26, 125-132.

North, J. B., and Jennett, S. (1974). Abnormal breathing patterns associated with acute brain damage. Archives of Neurology, 31, 338-344.
Pampiglione, G. (1956). Some anatomical considerations upon electrode placement in routine EEG. Proceedings of Electrophysiological Technologists' Association, 7, 20-30.

Pavlov, J. (1927). Conditioned Reflexes, an Investigation of the Physiological Activity of the Cerebral Cortex. Oxford University Press: London.

Poole, E. W. (1960). Periodic EEG discharges in subacute encephalitis with reference to respiratory and cardiac cycles. Electroencephalography and Clinical Neurophysiology, 12, 759.

Robin, E. D., Whaley, R. D., Crump, C. H., and Travis, D. M. (1957). Alveolar gas concentrations and respiratory center sensitivity to $\mathrm{CO}_{2}$ during natural sleep. Federation Proceedings, 16, 107-108.

Sokolov, E. N. (1960). Neuronal models and the orienting reflex. In The Central Nervous System and Behavior, pp. 187-276. Edited by M. A. B. Brazier. Josiah Macy Jr Foundation: New York.

Sokolov, E. N., and Paramonova, N. P. (1961). Progressive changes in the orienting reflex in man during the development of sleep inhibition. Pavlov, J. Journal of Higher Nervous Activity, 11, 217-226.

Strauss, H. (1929). Das Zuzammenschrechen. Journal fïr Psychologie und Neurologie, 39, 111-321.

Symon, L., Dorsch, N. W. C., and Stephens, R. T. (1972). Pressure waves in so-called low pressure hydrocephalus. Lancet, 2, 1291. 